\title{
Analytical evaluation of breath acetone tubes
}

\author{
(C) Anastasia M. Ivanova,,$^{1,3} *^{+}$and Elena O. Kolomina ${ }^{2,3}$ \\ ${ }^{1}$ Department of Molecular Biotechnology. Saint-Petersburg State Institute of Technology. \\ Moskovsky Ave., 26. Saint-Petersburg, 190013. Russia. Phone: +7 (9312) 67-94-69. \\ E-mail: ivanovanastia91@mail.ru \\ ${ }^{2}$ Department of Analytical Chemistry. Saint Petersburg State University, Institute of Chemistry. \\ University Ave., 26. Petergof. Saint-Petersburg, 198504. Russia. \\ Phone: +7 (9111) 54-28-35. E-mail: lensnpe@gmail.com \\ ${ }^{3}$ AMA Co. Ltd. 17 line V.O. 4-6. Saint-Petersburg, 199034. Russia. \\ Phone:+7812-380-7699.E-mail:ai@amamed.ru,e_kolomina@amamed.ru
}

\begin{abstract}
*Supervising author; ${ }^{+}$Corresponding author
Keywords: metabolic flexibility, ketosis, ketone bodies, acetone, exhaled air, express diagnostics.
\end{abstract}

\begin{abstract}
Breath analysis provides opportunities for further development of precise and quick non-invasive diagnostic tools. The important example is the monitoring of metabolic flexibility through the acetone levels in an exhale. Metabolic changes may cause such pathological conditions as metabolic syndrome, type 2 diabetes mellitus and obesity. It is proven that breath acetone levels can indicate the states of ketosis or ketoacidosis. The development of sensitive, selective and easy-to-use tests for breath acetone is a step to personalized diagnostics, preliminary diagnosis and therapeutic control

The aim of the research was to evaluate analytical characteristics of acetone breath tubes intended for non-invasive monitoring of metabolic state.

The test is designed as an easy-to-blow glass tube, comprising a chemical reagent highly sensitive to acetone. The reagent changes its color from yellow to magenta depending on acetone concentrations. Sensitivity assessment was performed by lab simulation of an exhaled breath with various acetone concentrations. The acetone levels corresponded to a range associated with various metabolic conditions and were controlled by titrimetric method and a portable breath analyzer. Additionally, specificity to a target gas in the presence of water and ethanol vapors was assessed.

The results showed a correlation between the acetone concentration and the color gradients of the acetone sensitive reagent. The tubes show no reactions towards water and ethanol vapors.
\end{abstract}

\section{References}

[1] W. Miekisch, J.K. Schubert, G.F.E. Noeldge-Schomburg. Diagnostic potential of breath analysis - focus on volatile organic compounds. Clin. Chim. Acta. 2004. Vol.347. No.1-2. P.25-39.

[2] L.D. Bos, P.J. Sterk, S.J. Fowler. Breathomics in the setting of asthma and chronic obstructive pulmonary disease. J. Allergy Clin. Immunol. 2016. Vol.138. No.4. P.970-976.

[3] B.M. Ross, R. Babgi. Volatile compounds in blood headspace and nasal breath. J. Breath Res. 2017. Vol.11. No.4. P.046001.

[4] B.H. Goodpaster, L.M. Sparks. Metabolic Flexibility in Health and Disease. Cell Metab. 2017. Vol.25. No.5. P.1027-1036.

[5] Z. Wang, C. Wang. Is breath acetone a biomarker of diabetes? A historical review on breath acetone measurements. J. Breath Res. 2013. Vol.7. No.3. P.037109.

[6] V. Ruzsányi. Breath acetone as a potential marker in clinical practice. J Breath Res. 2017. P.19.

[7] B. Buszewski et al. Detection of volatile organic compounds as biomarkers in breath analysis by different analytical techniques. Bioanalysis. 2013. Vol.5. No.18. P.2287-2306.

[8] B. Buszewski et al. Human exhaled air analytics: biomarkers of diseases. Biomed. Chromatogr. 2007. Vol.21. No.6. P.553-566.

[9] J. King et al. A mathematical model for breath gas analysis of volatile organic compounds with special emphasis on acetone. J. Math. Biol. 2011. Vol.63. No.5. P.959-999.

[10] A. Rydosz. A Negative Correlation Between Blood Glucose and Acetone Measured in Healthy and Type 1 Diabetes Mellitus Patient Breath. J. Diabetes Sci. Technol. 2015. Vol.9. No.4. P.881-884. 
[11] M. Fleischer et al. Detection of volatile compounds correlated to human diseases through breath analysis with chemical sensors. 2002. P.5.

[12] M.B. Greiter et al. Differences in Exhaled Gas Profiles Between Patients with Type 2 Diabetes and Healthy Controls. Diabetes Technol. Ther. 2010. Vol.12. No.6. P.455-463.

[13] W. Lindinger, A. Hansel, A. Jordan. On-line monitoring of volatile organic compounds at pptv levels by means of proton-transfer-reaction mass spectrometry (PTR-MS) medical applications, food control and environmental research. Int. J. Mass Spectrom. Ion Process. 1998. Vol.173. No.3. P.191-241.

[14] N. Teshima et al. Determination of acetone in breath. Anal. Chim. Acta. 2005. Vol.535. No.1-2. P.189-199.

[15] KETONIX®, BLUETOOTH, Basic Manual Version 1.1 [Электронный pecypc]:

https://fellrnr.com/wiki/Breath_Acetone_Meters, https://www.ketonix.com/images/PDF/Basic-ManualKetonix-Bluetooth-EN-v1.1.pdf, https://www.ketonix.com/support-technique\#sppb-tab1-2, https://www.ketonix.com/support-interpretation 